\title{
Inhibición de la relajación plástica en heteroestructuras InGaAs/GaAs(001) crecidas a baja temperatura
}

\author{
M. HERRERA, D. GONZÁLEZ, M. U. GONZÁLEZ*, Y. GONZÁLEZ*, L. GONZÁLEZ*, R. GARCÍA \\ Departamento de Ciencia de los Materiales e I.M. y Q.I., U. de Cádiz, Apartado 40, 11510 Puerto Real, Cádiz, España \\ *Instituto de Microelectrónica de Madrid, C/ Isaac Newton, 8, 28760-Tres Cantos, Madrid, España
}

\begin{abstract}
En este trabajo se han estudiado capas simples de InGaAs $/ \mathrm{GaAs}(001)$ crecidas a $200^{\circ} \mathrm{C}$ y $500^{\circ} \mathrm{C}$ por Microscopía Electrónica de Transmisión (TEM) y Difracción de Rayos X de Doble Cristal (DCXRD). Los resultados obtenidos muestran que el crecimiento a baja temperatura inhibe el proceso de relajación plástica, apareciendo las dislocaciones de desajuste (DD) durante el ciclo térmico posterior al crecimiento. El grado de relajación plástica final alcanzado en las muestras recocidas es inferior respecto de las crecidas a alta temperatura, debido a la existencia de una sola superficie donde nuclear dichas dislocaciones. Además, se ha observado que la modulación de composición también dificulta la relajación plástica, ya que introduce puntos de tensión en el seno del material que obstaculizan el movimiento de las dislocaciones, tanto en estructuras crecidas a alta como a baja temperatura.
\end{abstract}

Palabras clave: inhibición de la relajación, crecimiento a baja temperatura, modulación de composición, InGaAs/GaAs(001), TEM

Plastic relaxation inhibition in low temperature growth of InGaAs/GaAs(001) heterostructures

Low and high temperature grown InGaAs/GaAs(001) epilayers have been studied by Transmission Electron Microscopy and Double Crystal X Ray Diffraction. Our results show that low temperature growth inhibits plastic relaxation, and misfit dislocations only appearing in the subsequent thermal annealing. The final plastic relaxation degree reached in this way is lower than in high temperature growth, due to the availability of a single surface where misfit dislocations could nucleate. In addition, we have observed that composition modulation even delays plastic relaxation flow, since it introduces tension points that block the dislocation movement, causing the strain-hardening of the alloy.

Keywords: relaxation inhibition, low temperature growth, composition modulation, In GaAs/GaAs(001), TEM

\section{INTRODUCCIÓN}

Para el óptimo desarrollo de la tecnología optoelectrónica resulta fundamental el disponer de materiales semiconductores cuyo ancho de banda prohibida (band-gap) pueda adaptarse a las necesidades de los nuevos dispositivos. El crecimiento de heteroestructuras semiconductoras conlleva serias dificultades debido a las diferencias entre sus parámetros reticulares, que pueden dar lugar a la formación de defectos cristalinos o inhomogeneidad en la composición. En este trabajo se realiza un estudio del estado de relajación y microestructura de capas de InGaAs $/ \mathrm{GaAs}(001)$ crecidas a $200^{\circ}$ y $500^{\circ} \mathrm{C}$. Los resultados revelan una deficiencia en el desarrollo de la relajación plástica de estas epicapas crecidas a baja temperatura. La formación de dislocaciones en la superficie, así como la presencia de modulación de composición (MC), se apuntan como los motivos de esta inhibición de la relajación plástica.

\section{MATERIALES Y MÉTODO}

Los sistemas considerados están constituidos por capas simples de $\mathrm{In}_{0.2} \mathrm{Ga}_{0.8} \mathrm{As} / \mathrm{GaAs}(001)$ obtenidos mediante Epitaxia por Haces Moleculares (MBE) a $500^{\circ} \mathrm{C}$ y Epitaxia por Haces Moleculares de Capa Atómica (ALMBE) a $200^{\circ} \mathrm{C}$. El estudio realizado comprende cinco estructuras, dos crecidas por MBE, B50 y B190, y tres obtenidas mediante ALMBE, A80Ct, A300 y A300Ct. El número de la muestra especifica el espesor en nanómetros de la epicapa y la nomenclatura " $\mathrm{Ct}^{\prime}$ " indica que la muestra en cuestión sufrió un ciclo térmico de $500^{\circ} \mathrm{C}$ posterior al crecimiento. Las muestras han sido estudiadas por Microscopía Electrónica de Transmisión (TEM) en un JEOL 1200 EX operando a $120 \mathrm{KV}$ en modo contraste de difracción y por Difracción de Rayos X de Doble Cristal.

\section{RESULTADOS EXPERIMENTALES}

En la Figura 1 se presentan los resultados de deformación residual en función del espesor en las muestras bajo estudio. Cabe destacar que A80Ct y A300Ct, dos muestras crecidas en iguales condiciones (baja temperatura y ciclo térmico) pero con una gran diferencia en el espesor de epicapa, tengan un grado de relajación bastante similar, alrededor de un 30\%. Además, B190, de espesor intermedio entre ellas (aunque crecida a $500^{\circ}$ ), aparece como la estructura más relajada de todas, con un porcentaje de relajación cercano al 55\%. También resalta la asimetría encontrada en $\mathrm{A} 300 \mathrm{Ct}$, en contraste con todas las demás estructuras, bastantes simétricas en relajación. Por otro lado, tanto B50 como A300 permanecen coherentes al substrato, lo que sería predecible para el caso de la primera, de bajo espesor, aunque no para la segunda, con capa de $300 \mathrm{~nm}$, lo que muestra que el crecimiento a baja temperatura inhibe la relajación.

En las micrografías obtenidas por TEM con la reflexión 220 para las estructuras crecidas a $200^{\circ} \mathrm{C} \mathrm{A} 300$ y A300Ct aparecen ciertos contrastes verticales claros y oscuros no observables con la reflexión 400, asociados a una modulación composicional debida a un proceso de descomposición espinodal $(1,2)$. En estas muestras, se observa la peculiaridad de que la longitud de onda de dichos contrastes es asimétrica y similar en las dos estructuras: unos $25 \mathrm{~nm}$ en la dirección [110] (modulación de contraste fino) y aproximadamente 60-70 nm en [1̄10] (modulación de contraste grueso), como se muestra en las micrografías de la figura 2 correspondientes a A300Ct. En general, se puede considerar que la modulación de contraste grueso es un artefacto de la preparación de muestra para TEM causado por las tensiones inducidas por la modulación fina ( ${ }^{3}$ ). Sin embargo, la estructura A80Ct, también crecida a baja temperatura, presenta una epicapa homogénea en contraste, a diferencia de las anteriores. Por otro lado, en las muestras B50 y B190, 
obtenidas a $500^{\circ} \mathrm{C}$ por $\mathrm{MBE}$, la $\mathrm{MC}$ es simétrica respecto a ambas direcciones $\langle 110\rangle$, presentando una longitud de onda de aprox. $25 \mathrm{~nm}$, similar a la observada en la dirección [110] de A300 y A300Ct.

\section{DISCUSIÓN}

En la figura 1 se representa la ley de relajación de Dunstan et al. $\left({ }^{4}\right)$, que presenta un excelente acuerdo con los resultados experimentales para predecir el grado de relajación plástica de heteroestructuras semiconductoras InGaAs/GaAs crecidas a temperaturas convencionales. Como se puede observar, $\mathrm{A} 300 \mathrm{Ct}$, con un ciclo térmico de $500^{\circ} \mathrm{C}$, tiene un grado de relajación muy superior a A300, deduciéndose que las DD encontradas deberán haberse formado durante el recocido. Aún así, el grado de relajación encontrado en esta estructura A300Ct es inferior al predicho por el modelo teórico y, además, es asimétrico. En comparación, B190, (crecida a $500^{\circ} \mathrm{C}$ ), presenta una relajación plástica simétrica y más en consonancia con el modelo.

La diferencia en relajación plástica observada en las distintas muestras es debida a que la relajación depende fuertemente de las condiciones de crecimiento. La muestra B190 se obtuvo a alta temperatura, de modo que suponemos que las dislocaciones se van formando progresivamente durante el crecimiento. Esto implica que dichas dislocaciones tienen "muchas superficies" para nuclear, conforme se va creciendo la muestra aparece una nueva superficie que puede producir dislocaciones que deslicen hasta la intercara, por lo que es predecible que esta muestra tenga un grado de relajación plástica considerable. En A300Ct, sin embargo, sólo existe una superficie para nuclear dislocaciones (la superficie final de la muestra), ya que las DDs aparecen en el ciclo térmico, por lo que la relajación se ve más dificultada que en el crecimiento a alta temperatura, y la deformación elástica final de la muestra será mayor.

Por otro lado, la MC podría influir también en la inhibición de la relajación plástica de las estructuras consideradas. Esto se deduce a partir de los resultados obtenidos para A80Ct y A300Ct, dos muestras crecidas en iguales condiciones, donde la estructura no modulada A80Ct se ajusta al modelo teórico, pero A300Ct, con $\mathrm{MC}$, presenta una relajación deficiente. La aparición de $\mathrm{MC}$ en un material provoca la deformación elástica de determinados planos atómicos, los cuales resultarán tensados. Estos puntos de tensión en el seno de la aleación pueden dificultar el deslizamiento de las dislocaciones formadas en el proceso de relajación plástica, de modo que el sistema sufriera un endurecimiento mecánico. De este modo, en la muestra homogénea A80Ct, las dislocaciones tienen relativa facilidad para deslizar, de modo que se alcanza una relajación considerable, a diferencia de A300Ct y todas las demás estructuras bajo estudio, donde la MC obstaculiza el movimiento de las dislocaciones, impidiendo que se alcance el grado de relajación predicho por Dunstan.

El endurecimiento mecánico del material causado por la existencia de MC se corrobora por la asimetría encontrada en la muestra A300Ct tanto en MC como en relajación. Así, paralelamente a la dirección con modulación fina se puede medir una densidad de dislocaciones bastante baja, de $7.5 \cdot 10^{4} \mathrm{~cm}^{-1}$, mientras que en la perpendicular, que tiene modulación gruesa, encontramos $19 \cdot 10^{4} \mathrm{~cm}^{-1}$. En referencia a esto, Cahn ${ }^{5}$ demostró que si el plano de deslizamiento o el vector de Burgers de una dislocación son paralelos o perpendiculares al vector de onda de la MC, las tensiones internas debidas a esta separación de fases no actúan sobre dicha dislocación y, por tanto, no existe endurecimiento mecánico. Según este razonamiento, las dislocaciones que aparecen en la figura 2.a, cuya línea de dislocación es paralela a la dirección [110] y deslizan por planos del tipo $\{111\}$, deberían sufrir la interacción con las tensiones internas de la estructura debidas a la existencia de la MC

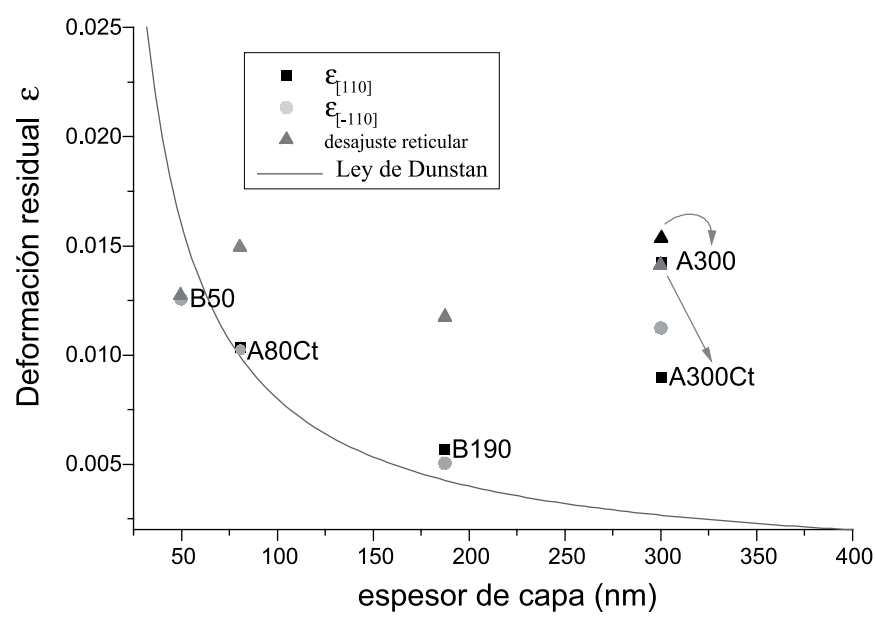

Fig. 1. Gráfica deformación residual, $\varepsilon$, -espesor de epicapa, donde se muestra la ley de Dunstan así como los resultados experimentales obtenidos para las estructuras estudiadas. . Se ha definido (insertar 04008_ECU_A ) y (insertar 04008_ECU_B ) siendo a y a los parámetros reticulares del sustrato y la epicapa, respectivamente y el superíndice 0 se refiere al estado libre de tensiones
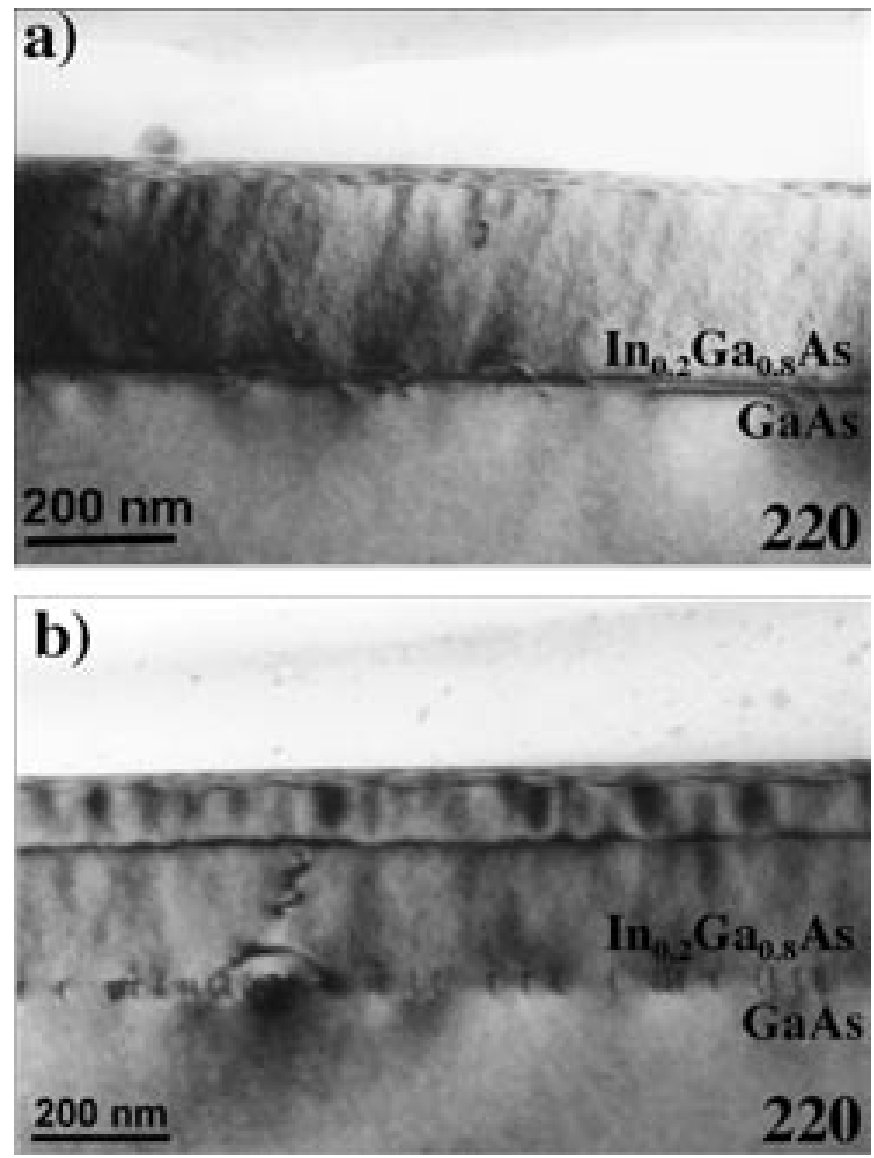

Fig. 2. Micrografías en sección transversal obtenidas con la reflexión 220 para la estructura A300Ct: (a) correspondiente a la dirección [110], (b) dirección [1]10]. Se observa una asimetría en la longitud de onda 
fina observada en esta sección de la muestra A300Ct. Sin embargo, y considerando que la modulación gruesa fuese un artefacto de la preparación de muestra para TEM, las dislocaciones de propagación que deslizan en la dirección [110] de esta estructura observadas en la figura 2.b no sufrirán ningún tipo de endurecimiento debido a la MC fina encontrada. Esta argumentación está en consonancia con nuestros resultados experimentales del grado de relajación plástica encontrado en esta estructura $\mathrm{A} 300 \mathrm{Ct}$, ya que la dirección con modulación fina presenta menor densidad de dislocaciones que la perpendicular $y$, además, apoyaría la hipótesis de que la modulación composicional provoca endurecimiento mecánico en un material. Por otro lado y en comparación, las demás estructuras estudiadas presentan MC fina en las dos secciones transversales, la cual causará un endurecimiento similar en ambas direcciones, e inducirá a que también la relajación sea simétrica, como muestran los resultados experimentales.

\section{CONCLUSIONES}

El estudio de capas InGaAs/ GaAs(001) crecidas a baja temperatura reflejó una deficiencia en el grado de relajación plástica alcanzado, debido a que la aparición de DD se produce en el ciclo térmico posterior y no durante el crecimiento como ocurre en estructuras crecidas a alta temperatura, con la consecuente dificultad debido a la existencia de una sola superficie donde nuclear dislocaciones. Por otro lado, se ha observado que la existencia de puntos de tensión en el material produ- cidos por la modulación composicional dificulta el movimiento de las dislocaciones en determinados planos, produciendo un endurecimiento mecánico de la aleación, independientemente de la temperatura de crecimiento utilizada.

\section{AGRADECIMIENTOS}

El presente trabajo está subvencionado por los proyectos de investigación MAT2000-1625, MAT2001-3362 y por la Junta de Andalucía (PAI TEP-0120).

\section{BIBLIOGRAFÍA}

1. K. Lee, B. A. Philips, R. S. McFadden y S. Mahajan. "Microstructural characteristics of mixed III-V epitaxial layers". Mat. Sci. and Eng. B, 32, 231-237 (1995).

2. G. Patriarche, F. Glass, G. Le Roux, L. Largeau, A. Mereuta, A. Ougazzaden y J. L. Benchimol. "TEM study of the morphological and compositional instabilities of InGaAsP epitaxial structures". J. Crystal Growth, 221, 12-19 (2000).

3. J. S. Choi, W. K. Choo, J. J. Lee y H. G. Lee. "The origin of coarse contrast modulation in $\mathrm{In}_{0.525} \mathrm{Ga}_{0.475}$ As layer on $\mathrm{InP}$ grown by MBE at $310^{\circ} \mathrm{C}^{\prime \prime}$. Mat. Lett., 32, 1-4 (1997).

4. D. J. Dunstan, S. Young, y R. H. Dixon. "Geometrical theory of critical thickness and relaxation instrained-layer growth". J. Appl. Phys, 70, 3038-3045 (1991).

5. J. W. Cahn. "Hardening by spinodal decomposition”. Acta Metallurgica, 11, 1275-1282 (1963).

Recibido: 1.2.03

Aceptado: 30.11 .03 